\title{
Peridural Torácica Alta Associada ou não à Peridural Torácica Baixa em Pacientes Ambulatoriais: Implicações Clínicas *
}

\section{High Thoracic Epidural Anesthesia Associated or not to Low Thoracic Epidural Anesthesia in Outpatient Procedures: Clinical Implications}

\author{
Djalma Sperhacke ${ }^{1}$; Karl Otto Geier ${ }^{2}$; João Carlos Correia Eschiletti ${ }^{3}$
}

\section{RESUMO}

Sperhacke D, Geier KO, Eschiletti JCC - Peridural Torácica Alta Associada ou não à Peridural Torácica Baixa em Pacientes Ambulatoriais: Implicações Clínicas

JUSTIFICATIVA E OBJETIVOS: Sob bloqueio peridural torácico baixo ou médio, as alterações hemodinâmicas são facilmente controladas. Como o bloqueio peridural torácico alto $\left(T_{2}-T_{3}\right)$ acomete, freqüentemente, as raízes do plexo braquial $\left(C_{4}\right) C_{5}-T_{1}\left(T_{2}\right)$, algumas destas responsáveis pela formação do nervo frênico $\left(C_{3}-C_{4}-C_{5}\right)$ é de se supor, possiveis repercussões motoras deste último. O presente estudo realizado em cirurgias estéticas, sob bloqueio peridural segmentar isolado em $T_{2}-T_{3}$ ou associado ao bloqueio peridural segmentar em $T_{11}-T_{12}$, avaliou as repercussões motoras na dinâmica respiratória assim como nos membros superiores e inferiores.

MÉTODO: Trinta e duas pacientes, estado físico ASA I e II, sem doença pulmonar broncoespástica, em atividade e peso corporal igual ou superior a $50 \mathrm{~kg}$, foram submetidas a 21 bloqueios peridurais torácicos isolados em $T_{2}-T_{3}$ e as 11 restantes, a bloqueios combinados em $T_{11}-T_{12}$, com ropivacaína a 7,5\% (45 a $90 \mathrm{mg}$ ) associada ao sufentanil (10 a $20 \mu \mathrm{g})$. Repercussões hemodinâmicas, respiratórias e motoras nos membros superiores e inferiores foram avaliadas respectivamente, sob monitorização não-invasiva, espirometria, força de preensão da mão e escala de Bromage.

RESULTADOS: A média de duração das cirurgias mamárias foi de 105 minutos com depressão motora dos membros superiores ( $p<$ 0,001), com recuperação motora acontecendo aos 117,2 $\pm 51,3$ minutos e a primeira manifestação de dor aos $485 \pm 221,2$ minutos. As cirurgias combinadas, que tiveram uma duração média de 165 minutos, com depressão motora dos membros inferiores grau 1 , em $40 \%$ e grau 2, em $60 \%$ das pacientes pela escala de Bromage, sua recuperação ocorreu aos $223,9 \pm 57,1$ minutos e a primeira manifestação de dor em repouso, aos $555 \pm 197,9 \mathrm{~min}$. As funções pulmonares, VEF1 (l/seg); PFE (I. $\left.\mathrm{min}^{-1}\right)$; CVF (litros) apresentaram-se alteradas respectivamente, em 15,20\% ( $p<$ $0,009) ; 13,36 \%(p<0,029)$ e 18,09\% $(p<0,007)$, com elevação de $8,75 \%$ do VEF1/CVF $(p<0,162)$. Hipotensão arterial $(\leq 30 \%$ dos valores iniciais) e bradicardia ( $\leq 55 \mathrm{bpm})$ ocorreram em cinco pacientes e tremores durante os bloqueios, em treze pacientes.

${ }^{*}$ Recebido da (Received from) Clínica Rio Branco de Porto Alegre, $R S$

1. Anestesiologista da Clínica Rio Branco

2. Anestesiologista do Hospital Municipal de Pronto Socorro de Porto Alegre/RS; Anestesiologista Colaborador da Clindor do Hospital São Lucas da PUC/RS; Mestre em Cirurgia pela UFRGS

3. Cirurgião Plástico da Clínica Rio Branco

Apresentado (Submitted) em 21 de julho de 2003

Aceito (Accepted) para publicação em 06 de novembro de 2003

Endereço para correspondência (Correspondence to)

Dr. Karl Otto Geier

Rua Cel. Camisão, 172

90540-030 Porto Alegre, RS

E-mail:karlotto@terra.com.br

(c) Sociedade Brasileira de Anestesiologia, 2004
CONCLUSÕES: Sob bloqueio torácico alto ou cérvico-torácico com doses e volumes reduzidos de soluções anestésicas, ocorrem repercussões motoras nos membros superiores e nas funções pulmonares. No entanto, a julgar por observações preliminares e do presente estudo, as alterações espirométricas foram estatisticamente significativas, porém, sem expressão clínica na dinâmica respiratória, sendo essencialmente decorrentes da paralisia dos nervos intercostais, mais que do nervo frênico.

Unitermos: ANESTESIA Ambulatorial; TÉCNICAS ANESTÉSICAS, Regional: peridural torácica

\section{SUMMARY}

Sperhacke D, Geier KO, Eschiletti JCC - High Thoracic Epidural Anesthesia Associated or not To Low Thoracic Epidural Anesthesia in Outpatient Procedures: Clinical Implications

BACKGROUND AND OBJECTIVES: Hemodynamic changes are easily controlled under low or median thoracic epidural block. Since high thoracic epidural block $\left(T_{2}-T_{3}\right)$ often affects brachial plexus roots $\left(C_{4}\right) C_{5}-T_{1}\left(T_{2}\right)$, some of them responsible for phrenic nerve formation $\left(C_{3}-C_{4}-C_{5}\right)$, potential motor repercussions on this nerve are to be expected. Our study performed during cosmetic surgeries under isolated segmental epidural block in $T_{2}-T_{3}$ or associated to segmental epidural block in $T_{11}-T_{12}$, has evaluated motor repercussions on respiratory dynamics, upper and lower limbs.

METHODS: Participated in this study 32 patients physical status ASA I and II, without active bronchospastic pulmonary disease and body weight equal to or above $50 \mathrm{~kg}$, 21 of whom were submitted to isolated thoracic epidural blocks in $T_{2}-T_{3}$ and the remaining patients (11) were submitted to a combined thoracic epidural blocks in $T_{11}-T_{12}$ with $7.5 \%$ ropivacaine (45 to $90 \mathrm{mg}$ ) associated to sufentanil (10 to $20 \mu \mathrm{g})$. Hemodynamic, respiratory and upper and lower limbs motor repercussions were evaluated by noninvasive monitoring, spirometry hand grasping strength and Bromage score, respectively.

RESULTS: Mean mammary surgeries duration was 105 minutes with upper limbs motor depression ( $p<0.001)$, with motor recovery at $117.2 \pm 51.3 \mathrm{~min}$ and first pain complaint at $485 \pm 221.2 \mathrm{~min}$. Combined surgeries with mean duration of $165 \mathrm{~min}$, with lower limbs motor depression level 1 in $40 \%$ and level 2 in $60 \%$ of patients according to Bromage score, with recovery at 223.9 \pm 57.1 min and first pain complaint at rest at $555 \pm 197.9 \mathrm{~min}$. Pulmonary functions VEF1 (L/sec); PFE (L. min $\left.{ }^{-1}\right)$; FVC (liters) were changed in $15.20 \%$ $(p<0.009) ; 13.36 \%(p<0.029)$ and $18.09 \%(p<0.007)$, respectively, with $8.75 \%$ VEF1/FVC increase $(p<0.162)$. There has been hypotension ( $\leq 30 \%$ above baseline values) and bradycardia $(\leq 55$ $\mathrm{pbm}$ ) in 5 patients and shivering during blockade in 13 patients.

CONCLUSIONS: Under high thoracic or cervico-thoracic blockades with decreased anesthetic solution doses and volumes, there have been upper limbs and pulmonary functions motor repercussions. However, according to preliminary observations and this study, spirometric changes were statistically significant, but without clinical expression in respiratory dynamics, being essentially more a consequence of intercostal nerves than of phrenic nerve paralysis.

Key Words: ANESTHESIA, Ambulatorial; ANESTHETIC TECHNIQUES, Regional: thoracic epidural 


\section{INTRODUÇÃO}

$A^{\mathrm{s}}$ s mastectomias e toracoplastias sob bloqueio peridural torácico foram realizadas há mais de meio século ${ }^{1}$. Desde então, poucos estudos sobre o tema foram divulgados. Além de Figueiredo ${ }^{1}$, outros trabalhos nacionais esboçaram uma anestesia peridural segmentar utilizando baixos volumes anestésicos destinados a procedimentos cirúrgicos cérvico-braquiais ${ }^{2}$ e a cirurgias plásticas tóraco-abdominais ${ }^{3}$. Contudo, quando não se objetiva a anestesia peridural segmentar, grandes volumes de anestésicos são necessários, mormente em cirurgias associadas ${ }^{4}$. Todos ${ }^{1-4}$ enfatizaram as alterações hemodinâmicas, demonstrando ser possível o seu controle. Entretanto, o comprometimento motor tóraco-abdominal repercutindo nas funções pulmonares, foi avaliado apenas numa pequena série de pacientes submetidos à cirurgias da parede abdominal alta, mediante bloqueio peridural no terço inferior torácico $\left(\mathrm{T}_{8}-\mathrm{T}_{9}\right)^{5}$. Como o bloqueio peridural torácico alto acomete, freqüentemente, as raízes do plexo braquial $\left(\mathrm{C}_{4}-\mathrm{C}_{5}-\mathrm{T}_{1}-\mathrm{T}_{2}\right)$, algumas destas responsáveis pela formação do nervo frênico $\left(\mathrm{C}_{3}-\mathrm{C}_{4}-\mathrm{C}_{5}\right)$, é de se supor possíveis repercussões motoras neste último. No entanto, avaliações espirométricas da dinâmica respiratória indicaram testes pulmonares inalterados ou pouco diminuídos ${ }^{6}$. O presente estudo, realizado em cirurgias estéticas femininas, sob bloqueio peridural segmentar em $\mathrm{T}_{2}-\mathrm{T}_{3}$ e em $\mathrm{T}_{11}-\mathrm{T}_{12}$, com baixo volume de ropivacaína a 7,5\% acrescido de sufentanil avaliou, objetivamente, as repercussões motoras nos membros superiores e membros inferiores ${ }^{7} \mathrm{e}$, indiretamente, a musculatura respiratória pelos testes espirométricos das funções pulmonares.

\section{MÉTODO}

Com a aprovação Institucional e o consentimento de 32 pacientes adultas, estado físico ASA I e II, sem doença pulmonar broncoespástica, em atividade e peso corporal igual ou superior a $50 \mathrm{~kg}$, observou-se, em regime aleatório, contínuo e ambulatorial, o desempenho de 32 bloqueios peridurais distribuídos em 21 bloqueios isolados em $\mathrm{T}_{2}-\mathrm{T}_{3}$ e os restantes, combinados com 11 bloqueios em $T_{11}-T_{12}$. Com acesso venoso periférico por cateter $18 \mathrm{G}$ iniciou-se o fluxo contínuo de 15 a 20 ml. $\mathrm{kg}^{-1}$ de solução glicofisiológica e Ringer com lactato durante os primeiros 45 minutos, seguido por fluxo de manutenção para controle da estabilidade hemodinâmica. As monitorizações não-invasivas da atividade elétrica cardíaca (ECG contínuo com cinco eletrodos colocados no dorso das pacientes), freqüência cardíaca (bpm), pressão arterial sistólica (PAS), pressão arterial média (PAM), pressão arterial diastólica (PAD); saturação da oxihemoglobina $\left(\mathrm{SpO}_{2}\right)$ foram registrados a zero, 5, 15, 30, 60, 90, 120, $150 \mathrm{e}$ 180 minutos. Antes dos bloqueios anestésicos e na posição sentada, as pacientes eram devidamente instruídas para os testes espirométricos e motor dos membros superiores. Em ambos os casos, os valores obtidos nas últimas 19 pacientes, antes (valores controle/referência) e aos vinte minutos de bloqueio (valores de estudo) eram comparados. Nos bloqueios peridurais em $\mathrm{T}_{2}-\mathrm{T}_{3}$ foram observados, a partir de uma inspiração profunda, a) os graus de amplitude da expansão torácica alta em relação à expansão torácica baixa (grau 0 = inspiração profunda sem distorções entre a expansão torácica alta e baixa; grau 1 = inspiração profunda com leve distorção da expansão torácica alta, expansão torácica baixa normal; grau 2 = inspiração profunda com moderada distorção da expansão torácica alta, expansão torácica baixa normal; grau 3 = inspiração profunda com acentuada distorção da expansão torácica alta ou sem expansão torácica alta, leve distorção torácica baixa; grau 4 = apnéia), b) a função pulmonar com o espirômetro portátil Enhance VMI ${ }^{\circledR}$, da Clement Clarke International, Edinburgo e, c) os graus de envolvimento motor dos membros superiores, pela força da mão aplicada sobre um manguito de pressão Erka ${ }^{\circledR}$, Alemanha, de coluna de mercúrio previamente inflado, respectivamente a $40 \mathrm{mmHg}$, (grau 0 = força manual normal; grau 1 = força manual reduzida até $33 \%$ dos valores de controle; grau $2=$ força manual reduzida entre $33 \%$ e $66 \%$ dos valores de controle; grau 3 = força manual reduzida entre $66 \%$ e $100 \%$ dos valores de controle mas ainda com movimentação digital). Quando combinado com bloqueio peridural em $\mathrm{T}_{11}-\mathrm{T}_{12}$, as repercussões do bloqueio motor dos membros inferiores, quadrile região inguinal, foram avaliados pela escala de Bromage modificada $^{7}$.

Todas as pacientes foram submetidas a bloqueios peridurais segmentares, visando dose e volume reduzidos de anestésico local, na posição sentada, com os membros inferiores pendendo pela borda da mesa cirúrgica, os pés colocados sobre um apoio, cabeça flexionada com o queixo tocando a região esternal, antebraços apoiados sobre as coxas e os ombros amparados por uma auxiliar posicionada à sua frente, sob sedação grau 2 pela escala de Mackenzie ${ }^{8}$ com midazolam, por via venosa, titulado (1 a $3 \mathrm{mg}$ ). Três pacientes não cooperativas foram excluídas devido à sedação grau 3 . Depois de bloquear previamente os trajetos das punções $\left(T_{2}-T_{3}\right.$ e $\left.\mathrm{T}_{11}-\mathrm{T}_{12}\right)$, com lidocaína a $2 \%$, uma agulha Tuohy $18 \mathrm{G}$ era introduzida com punção mediana até ao espaço peridural, identificado pela perda de resistência ao ar. Enquanto os bloqueios isolados em $T_{2}-T_{3}$ destinavam-se à cirurgias plásticas de mama, com ou sem lipoaspirações úmidas (1 ampola de adrenalina em $400 \mathrm{ml}$ de solução fisiológica) axilares e nas faces medial e posterior do braço $\left(T_{1}-T_{2}\right)$, os bloqueios em $T_{11}-T_{12}$ destinavam-se aos procedimentos cirúrgicos combinados, compreendendo lipoaspirações abdominais altas e abdominoplastias ou lipoaspirações abdominais baixas, do trocanter $\left(L_{1}-L_{2}\right)$ e face medial das coxas $\left(L_{1}-L_{3}\right)$. Nestes casos, priorizava-se o acesso peridural $T_{11}-T_{12}$ com cateter $18 \mathrm{G}$, posicionado no espaço peridural até $4 \mathrm{~cm}$ respectivamente, no sentido cefálico nas cirurgias envolvendo metâmeros mais altos e, inversamente, no sentido caudal quando se visava metâmeros mais baixos. A permeabilidade do cateter era testada com um a dois mililitros da solução anestésica e, antes de fixá-lo sobre a pele até a região supraescapular, realizava-se o bloqueio em $\mathrm{T}_{2}-\mathrm{T}_{3}$. Na seqüência, após a colocação dorsal dos eletrodos, as pacientes eram posicionadas 
para a cirurgia, em decúbito supino, com o dorso levemente em cefaloaclive de $\leq 10^{\circ}$ e os membros inferiores enfaixados e elevados em posição de "canivete aberto", prevenindo represamento sangüíneo nas extremidades inferiores, a fim de manter o reflexo de Frank-Starling. Vinte minutos após, com o bloqueio instalado, um breve cefaloaclive de $45^{\circ}$ condicionava às pacientes nova aferição dos testes pulmonares. Nas cirurgias mamárias com ou sem lipoaspiração braquial ou axilar, a solução anestésica, em dose única, constou de ropivacaína a $7,5 \%$ (6 $\mathrm{ml}=45 \mathrm{mg})$ acrescido de sufentanil $(2 \mathrm{ml}=$ $10 \mu \mathrm{g}$ ), num volume total de $8 \mathrm{ml}$ e administrados de forma lenta e no sentido cefálico. No caso de cirurgias abdominais associadas, o volume da mesma mistura variou entre $12 \mathrm{ml} \mathrm{e}$ $14 \mathrm{ml}$ ( 75 a $90 \mathrm{mg}$ de ropivacaína a $7,5 \%$ e $10 \mu \mathrm{g}$ de sufentanil) em função do peso e administrados pelo cateter, aproximadamente 15 minutos antes do término das mamoplastias. Quando necessário, doses de reforço equivalente a $50 \%$ da dose inicial eram administradas nas cirurgias mais prolongadas, que ultrapassavam 200 minutos. A distribuição e o nível de sensibilidade dos dermátomos eram verificados pelo anestesiologista através da picada de agulha $27 \mathrm{G}$, nas mamoplastias, cada três minutos até a instalação final do bloqueio, aproximadamente aos 20 minutos, ou pelo cirurgião, com pinça cirúrgica nos metâmeros, compreendendo as intervenções associadas. Todas as cirurgias foram realizadas sob fluxo de 2 I. $\mathrm{min}^{-1}$ de $\mathrm{O}_{2}$ administrado por cateter nasal. Foram registrados os efeitos adversos dos bloqueios.

\section{RESULTADOS}

Os dados demográficos das pacientes $(n=32)$ e tipos de cirurgias estão na tabela I.

Tabela I - Dados Demográficos, Distribuição e Tipos de Cirurgias

\begin{tabular}{lc}
\hline Variáveis & \\
\hline Idade (anos) * $^{*}$ & $35,2 \pm 9,8(18-59)$ \\
Peso (kg) * & $58,9 \pm 7,2(51-81)$ \\
Estado físico * & \\
$\quad$ ASA I & $30(93,75 \%)$ \\
$\quad$ ASA II & $2(6,25 \%)$ \\
Mamoplastia isolada & 21 pacientes (2 exclusões) ** \\
$\begin{array}{l}\text { Mamoplastia e lipoaspiração } \\
\text { (axilar e MS) }\end{array}$ & 2 pacientes \\
$\begin{array}{l}\text { Mamoplastia e lipoaspiração } \\
\text { (abdominal) }\end{array}$ & 5 pacientes (1 exclusão) ** \\
$\begin{array}{l}\text { Mamoplastia e abdominoplastia } \\
\text { Mamoplastia, lipoaspiração } \\
\text { (abdominal) e lipoaspiração (MI) }\end{array}$ & 2 pacientes \\
* Valores expressos pela Média \pm DP \\
** Pacientes excluídas por não cooperarem com os testes realizados
\end{tabular}

A média de duração das cirurgias mamárias isoladas e associadas à lipoaspirações braquiais foi de 105 e 165 minutos, respectivamente, enquanto a regressão motora completa teve a média de 117,2 \pm 51,3 minutos (variação entre 105 e 130 minutos), ocorrendo a primeira manifestação de dor aos $485 \pm 221,2$ minutos (variação entre 400 e 820 minutos). Nas cirurgias combinadas, a regressão motora completa lombossacral pela escala de Bromage foi de 223,9 \pm 57,1 minutos (variação entre 198 e 327 minutos) e a primeira manifestação de dor em repouso, aos $555 \pm 197,9$ minutos (variação entre 203 e 645 minutos). Três pacientes receberam doses de reforço.

As alterações das funções pulmonares sob bloqueio peridural alto, estão na tabela II.

Tabela II - Funções Pulmonares sob Bloqueio Peridural $\mathrm{T}_{2}-\mathrm{T}_{3}$ em 19 Pacientes

\begin{tabular}{|c|c|c|c|c|}
\hline & $\begin{array}{l}\text { Pré-Bloqueio } \\
\text { (Controle) }\end{array}$ & $\begin{array}{c}20 \text { Minutos de } \\
\text { Bloqueio (Estudo) }\end{array}$ & $\begin{array}{c}\text { Variação } \\
(\%)\end{array}$ & $\begin{array}{c}\text { Valor de } \\
p\end{array}$ \\
\hline $\begin{array}{l}\text { VEF1 } \\
\text { I.seg }^{-1}\end{array}$ & $2,47(1,89-3,39)$ & $2,06(1,02-2,91)$ & $-16,60$ & 0,009 * \\
\hline $\begin{array}{l}\text { PFE } \\
\text { I. } \mathrm{min}^{-1}\end{array}$ & $2,53(2,18-3,96)$ & $2,23(1,32-3,05)$ & $-11,86$ & 0,029 * \\
\hline CVF - I & $2,43(2,03-4,18)$ & $2,31(1,67-3,83)$ & 4,94 & 0,007 * \\
\hline VEF1/CVF & 0,82 & 0,86 & $+4,87$ & 0,162 \\
\hline
\end{tabular}

Pico de Fluxo Expiratório (PFE - I. $\mathrm{min}^{-1}$ ); Volume Expiratório Forçado 1 seg (VEF1 - I.seg ${ }^{-1}$ ); Capacidade Vital Forçada (CVF - I); Índice de Tiffeneau (VEF1/CVF);

Aferidos com o espirômetro portátil Enhance $\mathrm{VMI}^{\circledR}$. Média e Amplitude em 19 pacientes;

* Estatisticamente significativo

O bloqueio peridural em $\mathrm{T}_{2}-\mathrm{T}_{3}$ de 29 pacientes (das 32 pacientes, três foram excluídas por falta de cooperação nos testes), registrou distribuição sensitiva assimétrica a partir de $\mathrm{C}_{8}(\mathrm{Fi}-$ gura 1). A perda de sensibilidade cranial foi desigual em duas pacientes nos metâmeros $\mathrm{C}_{7}$ e $\mathrm{C}_{6}$ com predominância em um lado e em três pacientes nos metâmeros $C_{5}$ e $C_{4}$. Ausência sensitiva caudal localizou-se uniformemente, em $\mathrm{T}_{6}-\mathrm{T}_{7}$.

Paralelamente, o acometimento motor dos membros superiores revelou, à direita pré-bloqueio (125,5 $\pm 21,9$ $\mathrm{mmHg}) /$ pós-bloqueio $(79,9 \pm 26,7 \mathrm{mmHg})$ e à esquerda, pré-bloqueio $(121,34 \pm 23,44 \mathrm{mmHg}) /$ pós-bloqueio $(85,4 \pm$ $26,9 \mathrm{mmHg}$ ) que resultaram clínica e estatisticamente significativos $(p<0,001)$. Os graus de intensidade motora pela

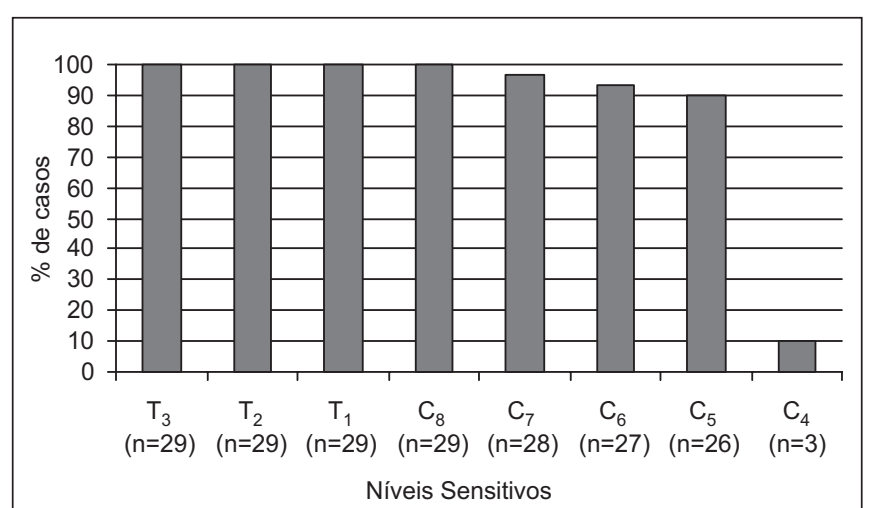

Figura 1 - Bloqueio $T_{2}-T_{3}$. Distribuição dos Metâmeros Sensitivos Cérvico-Torácicos 
Escala de preensão da mão foram: mão direita, grau $1=$ $15,8 \%$ ( 3 pacientes); grau $2=36,8 \%$ ( 7 pacientes $)$; grau $3=$ $47,4 \%$ (9 pacientes); mão esquerda, grau $1=31,6 \%$ ( 6 pacientes); grau $2=47,4 \%$ ( 9 pacientes $)$; grau $3=21 \%$ ( 4 pacientes $)$ ( Figuras 2, 3 e Tabela III).

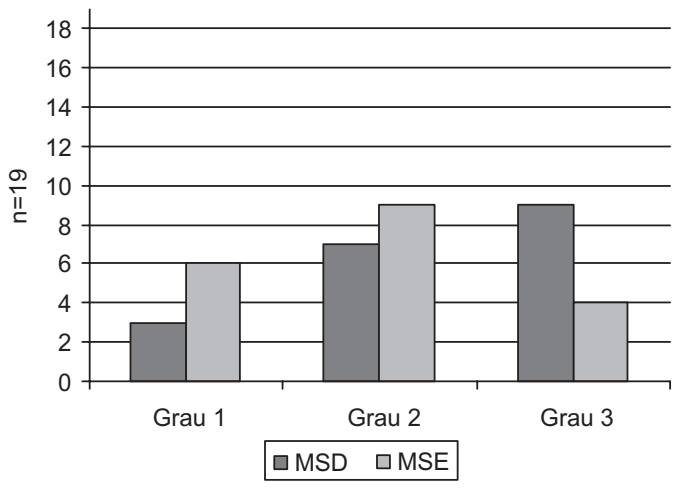

Figura 2 - Bloqueio Motor Braquial. Escala de Preensão da Mão

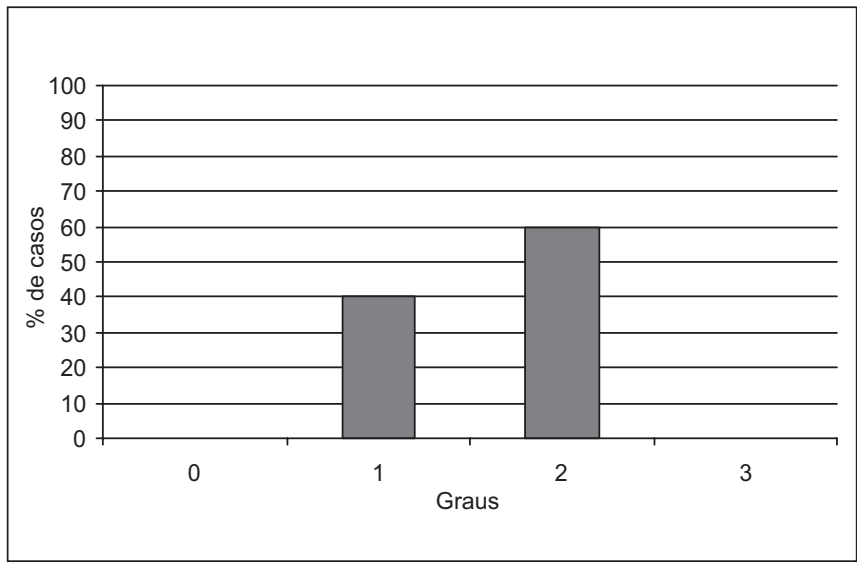

Figura 3 - Bloqueio Motor Lombossacral. Escala de Bromage

Tabela III - Bloqueio Motor Braquial 20 Minutos Pós-Bloqueio

\begin{tabular}{cccc}
\multicolumn{2}{c}{$(n=19){ }^{*}$} & & \\
\hline & Grau 1 & Grau 2 & Grau 3 \\
\hline MSD & 3 & 7 & $9^{* *}$ \\
MSE & 6 & 9 & $4^{* *}$ \\
\hline
\end{tabular}

* Aferido com esfigmomanômetro de mercúrio $E R K A^{\circledR}$ Alemanha;

** Dois pacientes mantinham movimentação digital, porém, sem força

Em função da orientação do cateter no espaço peridural, a dispersão sensitiva cranial do bloqueio $T_{11}-T_{12}$ atingiu ou sobrepôs aos limites inferiores do bloqueio $\mathrm{T}_{2}-\mathrm{T}_{3}$, e a dispersão caudal atingiu a região sacral. O bloqueio peridural motor em $\mathrm{T}_{11}-\mathrm{T}_{12}$ foi avaliado pela escala de Bromage modificada, registrando grau 1 ou parcial em quatro pacientes $(40 \%)$, e grau 2 ou semicompleto, em seis pacientes (60\%) (Figura 3 ). Os perfis oximétrico e hemodinâmico estão registrados nas figuras 4,5 e 6 e tabela IV.

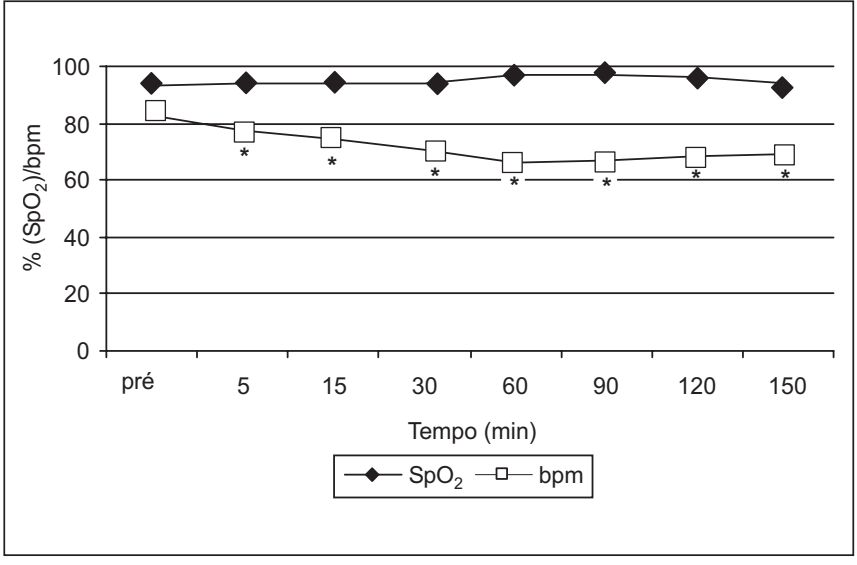

Figura 4 - Valores Médios Intra-Operatórios da Oximetria Arterial e Freqüência Cardíaca

* Estatisticamente significativo em relação aos valores de controle

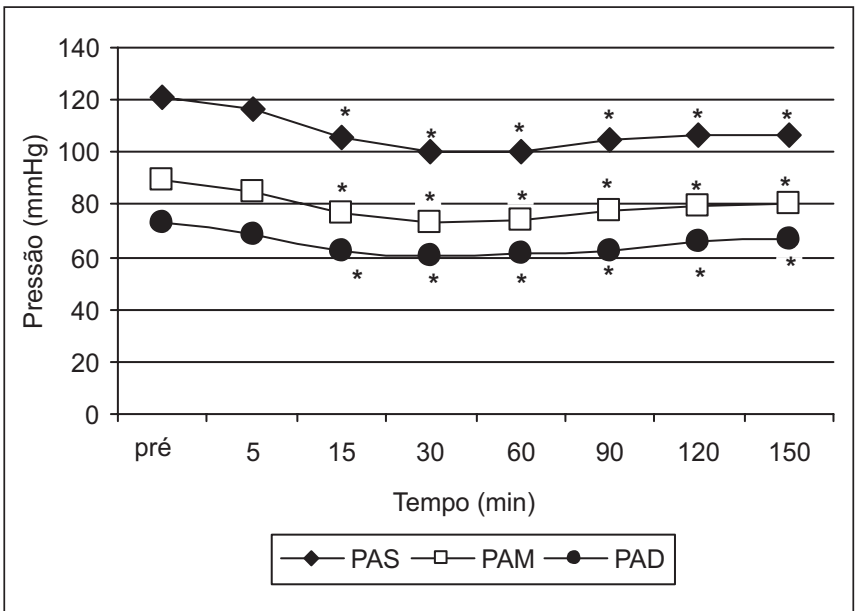

Figura 5 - Valores Peri-Operatórios Médios PAS, PAM, PAD * Estatisticamente significativo em relação aos valores de controle

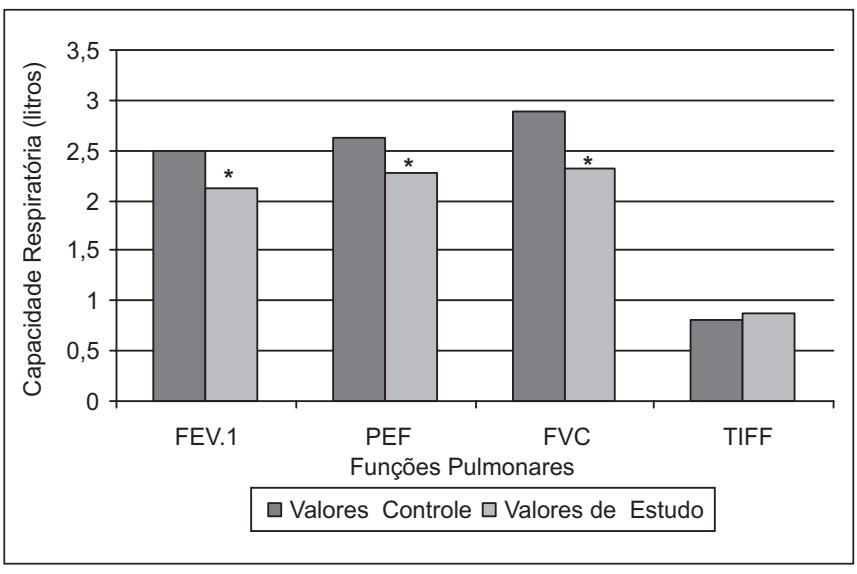

Figura 6 - Perfil Espirométrico das Médias das Funções Pulmonares Pré e aos 20 Minutos de Bloqueio

* Estatisticamente significativo aos pré-valores

Revista Brasileira de Anestesiologia Vol. 54, No 4, Julho - Agosto, 2004 
Tabela IV - Efeitos Adversos (Pacientes/\%)

\begin{tabular}{|c|c|}
\hline Sinal Claude Bernard Horner & $=4(12,5 \%)$ \\
\hline Bradicardia (freqüência $\leq 55 \mathrm{bpm}$ ) * & $=5(15,62 \%)$ \\
\hline $\begin{array}{l}\text { Hipotensão arterial (PAS } \leq 30 \% \text { do controle } \\
\text { ou PAM } \leq 50 \mathrm{mmHg} \text { ** }\end{array}$ & $=5(15,62 \%)$ \\
\hline Tremores & $=13(40 \%)$ \\
\hline $\begin{array}{l}\text { Desconforto durante os implantes } \\
\text { (próteses mamárias) }\end{array}$ & $=4(12,5 \%)$ \\
\hline Náuseas ${ }^{* \star *}$ / vômitos & $=5 / 0(15,6 \%)$ \\
\hline Prurido & $=1(3 \%)$ \\
\hline Dispnéia & $=1(3 \%)$ \\
\hline
\end{tabular}

* Tratado com 0,5 mg atropina

** Tratado com 5 a $15 \mathrm{mg}$ efedrina

*** Tratado com $4 \mathrm{mg}$ ondansetron

\section{Análise Estatística}

Os valores demográficos dos pacientes foram apresentados em média, desvio padrão e amplitude. Os dados obtidos pela escala de Bromage, os paraefeitos dos bloqueios peridurais e os testes espirométricos das funções pulmonares foram expressos em freqüências relativas, utilizando o teste $t$ de Student pareado para as variáveis FPE,VEF1, CVF e VEF1/CVF com o objetivo de avaliar as alterações dos parâmetros ventilatórios.

Para as variáveis categóricas da escala de Preensão da Mão antes e depois do bloqueio em $\mathrm{T}_{2}-\mathrm{T}_{3}$ foi utilizado o teste $t$ de Student pareado e para a comparação das médias hemodinâmicas (PAS, PAM, PAD e FC) antes e depois do bloqueio, o teste paramétrico ANOVA (Análise de Variância) para medidas repetidas, seguido do teste das diferenças mínimas significativas. O nível de significância foi estabelecido para um $\mathrm{p}<0,05$.

\section{DISCUSSÃO}

No Brasil, o bloqueio peridural se popularizou nas cirurgias estéticas mamárias ambulatoriais com ou sem sedação peri-operatória. Propofol em infusão contínua ${ }^{4}$ ou midazolam ${ }^{9}$ com ou sem fentanil são titulados para se obter graus de sedação em anestesia regional. Por ser o grau de sonolência um marcador na determinação da sedação, independente de sua etiologia farmacológica, utilizou-se a Escala de Sedação de Mackenzie ${ }^{8}$, para o midazolam. O grau de sedação ideal nas técnicas anestésicas regionais é a "sedação consciente" ${ }^{10}$ ou grau $2^{8}$, principalmente quando se necessita da cooperação do paciente para a realização de determinados testes de pesquisa.

Não obstante os dermátomos torácicos para mamoplastias estejam situados entre $T_{1}-T_{5}$, há a necessidade de incluir o dermátomo cervical $\mathrm{C}_{5}$ cuja raiz participa também na formação do nervo frênico. Em função da espessura das fibras nervosas, a anestesia regional com anestésicos locais sobre o neuroeixo é dissociativa, sendo mais cranial para as fibras $\beta$ (simpáticas), e mais caudal para as fibras $A \alpha$ (motoras) e, na posição intermediária, as fibras sensitivas. Especulam-se três razões para esse fenômeno: 1) maior resistência das fibras motoras em relação às fibras sensitivas, 2) insuficiente dispersão da solução anestésica em atingir, no mínimo, três nódulos consecutivos de Ranvier ${ }^{11}$ das raízes motoras localizadas no interior da "manga" dural, na altura dos foramens intervertebrais ${ }^{12} \mathrm{e}, 3$ ) susceptibilidade fisiológica das fibras nervosas aos anestésicos locais, decorrente da densidade dos canais de sódio e de potássio e da atividade metabólica das bombas iônicas ${ }^{12}$.

Algo semelhante deve ter acontecido na pesquisa motora da mão envolvendo os nervos radial $\left[C_{5}-\left(T_{1}\right)\right]$, mediano $\left[\left(C_{5}\right)-T_{1}\right]$ e ulnar $\left[\mathrm{C}_{7}-\left(\mathrm{T}_{1}\right)\right]$ e na flexão do antebraço através do músculo-cutâneo $\left(\mathrm{C}_{5}-\mathrm{C}_{7}\right)$. O ato de fechar a mão, envolvendo ou não objetos, apertando-os, assim como a capacidade de flexionar e estender o cotovelo, recruta os nervos referidos. A preensão exercida sobre um manguito de pressão previamente inflado a $40 \mathrm{mmHg}$ de uma coluna de mercúrio, aferida antes e aos vinte minutos de bloqueio instalado, informa, quantitativamente, a variação de atividade motora da mão. De um modo geral, os dermátomos anestesiados não se sobrepõem aos miótomos correspondentes. É o que acontece nos membros superiores, devido à distribuição periférica do plexo braquial e à farmacocinética da ropivacaína no espaço peridural.

Embora os bloqueios peridurais em $\mathrm{T}_{2}-\mathrm{T}_{3}$ do presente estudo tenham registrado perda de sensibilidade e $\mathrm{C}_{4}$, a dispersão cranial da solução anestésica no espaço peridural não foi uniforme a partir de $\mathrm{C}_{8}$, apresentando diferentes níveis de bloqueios sensitivo (Figura 1) e motor (Figura 2). Cranialmente, a partir de $\mathrm{C}_{7}$, o alargamento medular cervical, oriundo da maior densidade neural destinada aos membros superiores, resulta na diminuição do espaço peridural, podendo influenciar na dispersão da solução anestésica. Além do estreitamento do espaço peridural, existem outras causas ${ }^{14}$, embora haja divergência quanto ao papel da "plica mediana dorsalis" ${ }^{15}$, a banda conjuntiva entre a duramáter e o ligamento amarelo no espaço peridural que o divide em compartimentos ventral e dois pôstero-laterais ${ }^{16}$. Como a ropivacaína apresenta dissociação anestésica mais expressiva que a bupivacaína e, principalmente em relação à lidocaína, quando acrescida de sufentanil, de intensa propriedade lipofílica segmentar, pode se impregnar assimetricamente, no estreito espaço peridural cervical. Foi devido a essas propriedades farmacológicas que se fez a opção por ambas, prevendo uma anestesia metamérica de boa qualidade e de menor risco de depressão respiratória em relação à morfina hidrofílica.

Além do diafragma, os músculos esternocleidomastoideo $\left(\mathrm{C}_{2}-\mathrm{C}_{3}\right)^{17}$, escaleno médio $\left(\mathrm{C}_{3}-\mathrm{C}_{4}\right)^{17}$, anterior e posterior $\left(\mathrm{C}_{5}-\mathrm{C}_{8}\right)^{18}$, participam na inspiração profunda. Por outro lado, os nervos intercostais inferiores participam na inervação do diafragma ${ }^{17}$. Em função dessas particularidades, supõe-se que a integridade medular motora do contingente cervical, incluindo o nervo frênico, na presença de paralisia motora de todos os músculos intercostais, garantirá a continuidade respiratória e vice-versa ${ }^{19,20}$. No entanto, o bloqueio peridural 
cervical, segundo alguns pesquisadores ${ }^{6,21}$, determina certo comprometimento do nervo frênico com diminuição de $25 \%$ do VEF1 e $9 \%$, da $\mathrm{PaO}_{2}$, embora sem expressão clínica. Contrariamente, Wittich e col. ${ }^{22}$, realizando bloqueio peridural em $\mathrm{C}_{7}-\mathrm{T}_{1}$, com $18 \mathrm{ml}$ de bupivacaína a $0,5 \%$ ou mepivacaína a $1 \%$, em cirurgias de cabeça e pescoço, algumas delas estendendo-se ao tórax para mastectomias oncológicas, verificaram aumento do VEF1 em pacientes com doença broncopulmonar obstrutiva crônica. Da mesma forma, realizando traqueostomia na altura dos metâmeros $\mathrm{C}_{3}$ e $\mathrm{C}_{4}$ sob bloqueio peridural em $\mathrm{C}_{7}-\mathrm{T}_{1}$, com perda de sensibilidade em $\mathrm{C}_{2}$, mediante $6 \mathrm{ml}$ de lidocaína a 1,5\%, não se constatou enfraquecimento da ventilação espontânea ${ }^{22}$. Paralelamente, neste paciente, o teste do "aperto de mão" foi normal.

A pesquisa da depressão motora dos músculos intercostais pode ser avaliada pelo ato de tossir ${ }^{24}$, pela observação clínica da expansão do arcabouço torácico durante inspiração profunda, pela magnetometria da expansão torácica forçada ${ }^{25}$, pela eletromiografia, por amostras gasométricas ou testes espirométricos. A capacidade pulmonar total se obtém com uma inspiração máxima e ao expirá-la tão rápida e intensamente possível, num espirômetro portátil, obtém-se a Capacidade Vital Forçada (CVF). Nesta manobra expiratória, outras funções pulmonares podem ser aferidas simultaneamente, como o Volume Expiratório Forçado no primeiro segundo (VEF1) e o Pico de Fluxo Expiratório (PFE). O VEF1 tem maior reprodutibilidade por ser esforço dependente, não obstante o PFE ser também um bom indicador na fase inicial da expiração, onde os músculos intercostais internos $\left(T_{1}-T_{11}\right)$ e músculos abdominais $T_{6}-L_{1}$ participam ativamente. Ajulgar pelos testes gasométricos e espirométricos, o bloqueio peridural torácico alto não deve ser a razão para o desconforto respiratório. Embora a sensação de dispnéia possa $\operatorname{ser}^{5,21}$ ou não ${ }^{26,27}$ decorrente da discreta hipercapnia, o desconforto respiratório referido por poucos pacientes pode ser conseqüência da excitação dos receptores "J" das paredes alveolares, como resultado do acúmulo sangüíneo da vasodilatação pelo bloqueio simpático torácico, principalmente nas regiões dependentes ${ }^{28}$, ou pelo bloqueio parcial das fibras motoras dos músculos respiratórios com redução da dinâmica respiratória, fenômeno manifestado principalmente em pacientes ansiosos.

Adessaturação da hemoglobina depende, na maioria das vezes, da depressão respiratória, ocasionada por opióides pré-anestésico ou associado às soluções anestésicas peridural ou subaracnóidea, alterações da complacência toráci$\mathrm{ca}^{29}$, taquipnéia com baixo volume de ar corrente resultando em baixa ventilação alveolar efetiva e à concentração de $\mathrm{O}_{2}$ inalado. Como as pacientes do estudo estavam com oxigênio via cateter nasal durante os procedimentos cirúrgicos, os valores aferidos se mantiveram estáveis ou levemente superiores aos valores iniciais (Figura.4). Cabe salientar que a ansiedade e a sedação podem influenciar na oximetria. De fato, isto foi observado em algumas pacientes ansiosas e apreensivas que exibiam taquipnéia e valores oximétricos iniciais inferiores aos valores intra-operatórios mais elevados durante sedação "consciente" com midazolam.
Sob bloqueio peridural lombar, a persistência da atividade motora abdominal e dos membros inferiores é relevante, respectivamente, durante o trabalho de parto e na alta hospitalar em cirurgias ambulatoriais. No nosso estudo, priorizou-se a determinação da função motora nos territórios atingidos pelos bloqueios peridurais nos membros inferiores pela escala de Bromage ${ }^{7}$, por ser mais prática e factível do que a escala de Van Zundert e col. ${ }^{30}$, pois, de acordo com esta última, a partir do decúbito dorsal horizontal nem todos os pacientes conseguem flexionar o tronco aos membros inferiores.

Tremores musculares que surgiram durante o bloqueio, provavelmente não devem ter sido sinais de toxicidade da ropivacaína, devido à baixa dose utilizada, mas ao reflexo termorregulador, numa tentativa de o organismo de manter a temperatura corporal, contrapondo-se à dissipação de calor pela vasodilatação periférica do bloqueio simpático.

As alterações circulatórias causadas pela anestesia peridural torácica alta, estão relacionadas ao bloqueio simpático pela diminuição da freqüência (cronotropismo negativo) e pela redução da contractilidade (inotropismo negativo) cardíaco. Em voluntários calmos, dosagens plasmáticas de catecolaminas durante diversos níveis de bloqueio peridural $\left(\mathrm{T}_{8}, \mathrm{~T}_{4}, \mathrm{C}_{8}\right)$ não registraram diminuição significativa da norepinefrina, sugerindo que a simpatectomia química é apenas parcial ${ }^{31}$ e que a vasoconstrição compensatória de outros territórios, não atingidos pelo bloqueio nervoso, amenizam a diminuição da pressão arterial.

A alta ambulatorial pós bloqueio peridural implica na reversão do bloqueio motor dos membros acometidos, sem instabilidade residual nos membros superiores. A pesquisa da função motora da mão também consta como critério para alta hospitalar. Infelizmente, por não existir teste quantitativo sobre o acometimento motor dos membros superiores, foi substituído o teste subjetivo do "aperto de mão", pela adaptação da técnica de Bouaziz e col. ${ }^{32}$ na determinação da força muscular da mão. O controle motor dos membros inferiores, responsáveis pela estabilidade da postura ortostática, sem tontura, deve permitir a deambulação normal sem auxílio. Paralelamente, presença de micção espontânea, estabilidade hemodinâmica, tolerância à ingestão, ausência de náuseas e vômitos e analgesia pós-operatória, complementam os critérios de alta ${ }^{33}$.

Dois terços das raízes frênicas participam parcialmente, na formação plexular do membro superior, especialmente se este for pré fixado. Embora a maioria dos autores não tenha verificado possíveis repercussões motoras nos membros superiores sob bloqueio torácico alto ou cérvico- torácico com doses e volumes reduzidos de soluções anestésicas, estas realmente ocorrem, como ficou demonstrado, quantitativamente, no presente estudo, pelo teste do manguito de pressão com coluna de mercúrio. No entanto, a julgar por observações preliminares e do presente estudo, mínimas alterações espirométricas e sem repercussões clínicas são observadas sob bloqueio peridural torácico, sendo estas decorrentes mais da paralisia dos nervos intercostais e menos do nervo frênico, resultando na preservação da dinâmica respiratória. 


\section{AGRADECIMENTOS}

O autor agradece o auxílio da epidemiologista Tânia Hirakata e do Martin Geier.

\section{High Thoracic Epidural Anesthesia Associated or not To Low Thoracic Epidural Anesthesia in Outpatient Procedures: Clinical Implications}

Djalma Sperhacke, M.D.; Karl Otto Geier, M.D.; João Carlos Correia Eschiletti, M.D.

\section{INTRODUCTION}

Mastectomies and thoracoplasties under thoracic epidural block were firstly performed more than 50 years ago ${ }^{1}$. Since then, few studies on the subject have been published. In addition to Figueiredo ${ }^{1}$, other Brazilian studies have outlined segmental epidural anesthesia with low anesthetic volumes for cervico-brachial procedure ${ }^{2}$ and thoraco-abdominal plastic surgeries ${ }^{3}$. However, when segmental epidural anesthesia is not the objective, large anesthetic volumes are needed, especially for associated surgeries ${ }^{4}$. All authors ${ }^{1-4}$ emphasize hemodynamic changes and have shown that these can be controlled. Thoraco-abdominal motor involvement affecting pulmonary functions, however, has only been evaluated in a small series of patients submitted to high abdominal wall surgeries with epidural blockade of the lower thoracic third $\left(T_{8}-T_{9}\right)^{5}$. Since high thoracic epidural block often affects brachial plexus roots $\left(\mathrm{C}_{4}-\mathrm{C}_{5}-\mathrm{T}_{1}-\mathrm{T}_{2}\right)$, some of them responsible for phrenic nerve formation $\left(\mathrm{C}_{3}-\mathrm{C}_{4}-\mathrm{C}_{5}\right)$, potential motor repercussions in this nerve are to be expected. Spirometric evaluations of respiratory dynamics, however, have indicated unchanged or mildly decreased pulmonary tests ${ }^{6}$. This study, performed with female patients submitted to cosmetic surgeries under segmental epidural block in $T_{2}-T_{3}$ and in $T_{11}-T_{12}$ with low volume of $7.5 \%$ ropivacaine plus sufentanil has objectively evaluated motor repercussions on upper (UL) and lower (LL) limbs ${ }^{7}$ and, indirectly, on respiratory muscles by pulmonary functions spirometric test.

\section{METHODS}

After the Institution's approval and their consent, participated in this study 32 adult female patients, physical status ASA I and II, without active bronchospastic pulmonary disease, weighing $50 \mathrm{~kg}$ or above in whom the behavior of epidural blocks was randomly and continuously observed in outpatient setting. Blockades were distributed in 21 isolated blockades in $T_{2}-T_{3}$ and the 11 remaining blockades were combined with blockades in $T_{11}-T_{12}$. Peripheral venous access with $18 \mathrm{G}$ catheter was obtained and continuous 15 to 20 $\mathrm{mL} . \mathrm{kg}^{-1}$ saline solution with glucose and lactated Ringer's were infused for the first 45 minutes, followed by maintenance rate to control hemodynamic stability. Noninvasive monitoring consisted of cardiac electric activity (continuous ECG with 5 electrodes placed on patients' dorsum), heart rate (bpm), systolic blood pressure (SBP), mean blood pressure (MBP), diastolic blood pressure (DBP) and hemoglobin oxygen saturation $\left(\mathrm{SpO}_{2}\right)$, and was recorded at zero, 5, 15, 30, $60,90,120,150$ and 180 minutes. Before anesthetic block and in the sitting position, patients were informed about spirometric and upper limb motor tests. In both cases, values obtained for the last 19 patients before (control/reference values) and 20 minutes after blockade (study values) were compared. As from a deep breathe, the following were observed for epidural blocks in $\mathrm{T}_{2}-\mathrm{T}_{3}$ : a) levels of high thoracic expansion amplitude as compared to low thoracic amplitude (level 0 = deep breathe without distortions between high and low thoracic expansion; level 1 = deep breathe with mild high thoracic distortion and normal low thoracic expansion; level $2=$ deep breathe with moderate high thoracic distortion and normal low thoracic expansion; level 3 = deep breathe with marked high thoracic distortion or without high thoracic expansion and mild low thoracic distortion; level 4 = apnea); b) pulmonary function with portable Enhance $\mathrm{VMI}{ }^{\circledR}$ spirometer (Clement Clarke International, Edinburgh); and c) upper limbs motor involvement by hand grasping an Erka ${ }^{\circledR}$, Germany mercury column pressure cuff previously inflated to 40 $\mathrm{mmHg}$ (level $0=$ normal grasping; level $1=33 \%$ grasping decrease as compared to baseline; level $2=33 \%$ to $66 \%$ grasping decrease as compared to baseline; level $3=66 \%$ to $100 \%$ grasping decrease as compared to baseline but still with digital movements). When combined with epidural block in T${ }_{11}-T_{12}$, motor block repercussions on lower limbs, hip and inguinal region were evaluated by modified Bromage's score ${ }^{7}$. All patients were submitted to segmental epidural blocks aiming at decreasing local anesthetic dose and volume, in the sitting position with lower limbs pending outside the table, feet placed on a support, head bent with chin touching the externum, forearms over thighs and shoulders supported by a nurse positioned in front of them, under level 2 sedation according to Mackenzie's sedation score ${ }^{8}$ with intravenous titrated midazolam (1 to $3 \mathrm{mg}$ ). Three non-cooperative patients were excluded due to sedation level 3 . After previously blocking punctures pathway $\left(T_{2}-T_{3}\right.$ and $\left.T_{11}-T_{12}\right)$ with $2 \%$ lidocaine, an 18G Tuohy needle was introduced by median puncture until the epidural space, identified by loss of resistance to air. While isolated blockades in $T_{2}-T_{3}$ aimed at mammaplasties with or without tumescent axillary and arm posterior and medial faces $\left(T_{1}-T_{2}\right)$ liposuctions (1 epinephrine ampoule in $400 \mathrm{~mL}$ saline), $\mathrm{T}_{11}-\mathrm{T}_{12}$ blockades aimed at combined surgical procedures, with high abdominal liposuctions and abdominplasty or low abdominal liposuctions of trochanter $\left(L_{1}-L_{2}\right)$ and medial thigh region $\left(L_{1}-L_{3}\right)$. In those cases, epidural access $T_{11}-T_{12}$ with $18 \mathrm{G}$ catheter positioned in the epidural space up to $4 \mathrm{~cm}$, respec- 
tively in the cephalad direction for surgeries involving higher metamers and caudally for lower metamers was prioritized. Catheter patency was tested with 1 to $2 \mathrm{~mL}$ anesthetic solution and before fixing it on the skin until the suprascapular region, $T_{2}-T_{3}$ blockade was induced. In the sequence, after dorsal placement of electrodes, patients were placed in the supine position and slightly head up $\leq 10^{\circ}$ while lower limbs were wrapped and raised in the "open knife" position to prevent blood retention in lower extremities and to maintain Frank-Starling reflex. Twenty minutes after and with the blockade installed, a mild $45^{\circ}$ head up position would allow for new pulmonary test measurement. In mammary surgeries, with or without arm or axillary liposuction, single dose anesthetic solution was $7.5 \%$ ropivacaine $(6 \mathrm{~mL}=45 \mathrm{mg})$ plus sufentanil $(2 \mathrm{~mL}=10 \mu \mathrm{g})$, in a total volume of $8 \mathrm{~mL}$ and slowly administered. For associated abdominal surgeries, the volume of the same mixture has varied $12 \mathrm{~mL}$ to $14 \mathrm{~mL}$ ( 75 to 90 $\mathrm{mg}$ of $7.5 \%$ ropivacaine and $10 \mu \mathrm{g}$ sufentanil) as a function of weight, and was administered via catheter approximately 15 minutes after surgery completion. When needed, booster doses equivalent to $50 \%$ of the initial dose were administered for longer surgeries going beyond 200 minutes. Dermatomes level and sensitivity were evaluated by the anesthesiologist through $27 \mathrm{G}$ needle prick during mammaplasties, every 3 minutes until final blockade installation, approximately at 20 minutes, or by the surgeon with surgical clamp on metamers involved in combined interventions. All surgeries were performed under $2 \mathrm{~L}$. $\mathrm{min}^{-1}$ oxygen flow via nasal catheter. Blockade adverse events were recorded.

\section{RESULTS}

Patients' demographics data $(n=32)$ and types of surgery are shown in table I.

Table I - Demographics Data, Distribution and Types of Surgery

\begin{tabular}{|c|c|}
\hline Variables & \\
\hline Age (years) * & $35.2 \pm 9.8(18-59)$ \\
\hline Weight $(\mathrm{kg})$ * & $58.9 \pm 7.2(51-81)$ \\
\hline \multicolumn{2}{|l|}{ Physical status * } \\
\hline ASAI & $30(93.75 \%)$ \\
\hline ASA II & $2(6.25 \%)$ \\
\hline Isolated mammaplasty & 21 patients ( 2 exclusions) ** \\
\hline $\begin{array}{l}\text { Mammaplasty \& liposuction } \\
\text { (axillary \& MS) }\end{array}$ & 2 patients \\
\hline $\begin{array}{l}\text { Mammaplasty \& liposuction } \\
\text { (abdominal) }\end{array}$ & 5 patients ( 1 exclusion) $* *$ \\
\hline Mammaplasty and abdominoplasty & 2 patients \\
\hline $\begin{array}{l}\text { Mammaplasty, liposuction } \\
\text { (abdominal) \& liposuction (MI) }\end{array}$ & 2 patients \\
\hline
\end{tabular}

Mean duration of isolated mammary surgeries or combined with arm liposuctions was 105 and 165 minutes, respectively, while mean total motor recovery was $117.2 \pm 51.3$ minutes (105 to 130 minutes). First pain complaint was at $485 \pm 22.1$ minutes (400 to 820 minutes). In combined surgeries, total lumbosacral motor recovery by Bromage's scale was $223.9 \pm$ 57.1 minutes (198 to 327 minutes) and first pain complaint at rest at $555 \pm 197.9$ minutes (203 to 645 minutes). Three patients received booster dose.

Spirometric evaluations of respiratory dynamics under high thoracic epidural anesthesia are shwon below (table II).

Table II - Pulmonary Functions under Epidural Block $T_{2}-T_{3}$ in 19 Patients

\begin{tabular}{lcccc}
\hline & $\begin{array}{c}\text { Pre-Blockade } \\
\text { (Control) }\end{array}$ & $\begin{array}{c}\text { 20 Minutes after } \\
\text { Blockade (Study) }\end{array}$ & $\begin{array}{c}\text { Variation } \\
(\%)\end{array}$ & p value \\
\hline $\begin{array}{l}\text { FEV1 } \\
\text { L.seg }^{-1}\end{array}$ & $2.47(1.89-3.39)$ & $2.06(1.02-2.91)$ & -16.60 & 0.009 \\
EFP & $2.53(2.18-3.96)$ & $2.23(1.32-3.05)$ & -11.86 & 0.029 \\
L.min & & & & \\
FVC - L & $2.43(2.03-4.18)$ & $2.31(1.67-3.83)$ & 4.94 & 0.007 \\
FEV1/FVC & 0.82 & 0.86 & +4.87 & 0.162 \\
\hline
\end{tabular}

Expiratory Peak Flow (EPF - L.min ${ }^{-1}$ ); Forced Expiratory Volume in $1 \mathrm{sec}$ $\left(\right.$ FEV1 - L. seg ${ }^{-1}$ ); Forced Vital Capacity (FVC - L); Tiffeneau's Index (FEV1/FVC):

Measuredd with portable Enhance $\mathrm{VMI}^{\circledR}$ spirometer. Mean and amplitude in 19 patients;

Statistically significant

Epidural block in $\mathrm{T}_{2}-\mathrm{T}_{3}$ of 29 patients (from 32 patients, 3 were excluded for non cooperation during tests) has recorded asymmetric sensory distribution as from $\mathrm{C}_{8}$ (Figure 1). Loss of cranial sensitivity was unequal in two patients in metamers $\mathrm{C}_{7}$ and $\mathrm{C}_{6}$ with predominance in one side, and in 3 patients in metamers $\mathrm{C}_{5}$ and $\mathrm{C}_{4}$. Caudal sensory block was uniformly located in $\mathrm{T}_{6}-\mathrm{T}_{7}$.

In parallel, upper limbs motor block has revealed pre-blockade $(125.5 \pm 21.9 \mathrm{mmHg}) /$ post-blockade $(79.9=26.7 \mathrm{mmHg})$ to the right, and pre-blockade $(121.34 \pm 23.44 \mathrm{mmHg}) /$ post-blockade $(85.4 \pm 26.9 \mathrm{mmHg})$ to the left, which were clinically and statistically significant $(p<0.001)$. Motor intensity levels by grasping score were: right hand, level $1=15.8 \%$ (3 patients); level $2=$

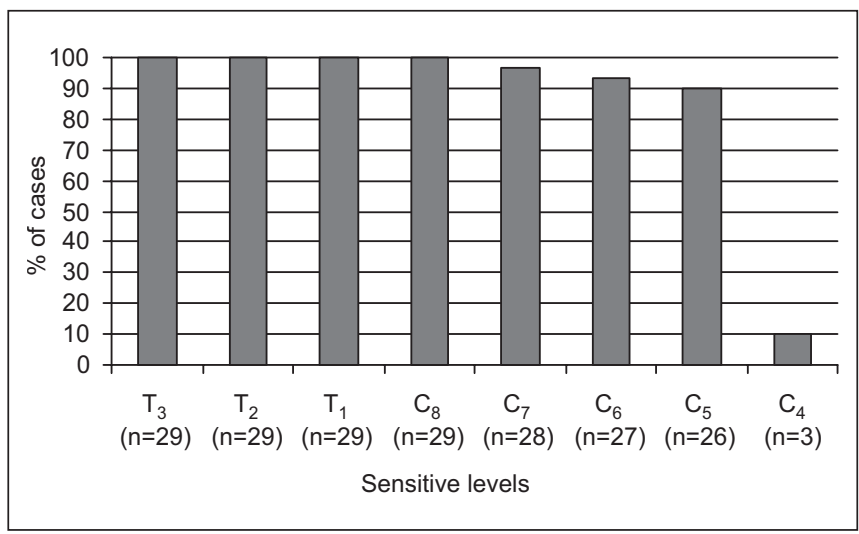

Figure $1-\mathrm{T}_{2}-\mathrm{T}_{3}$ Blockade. Distribution of Cervico-Thoracic Sensory Metamers 
$36.8 \%$ (7 patients); level $3=47.4 \%$ ( 9 patients); left hand, level 1 $=31.6 \%$ ( 6 patients); level $2=47.4 \%$ (9 patients); level $3=21 \%$ (4 patients) (Figures 2, 3 and Table III).

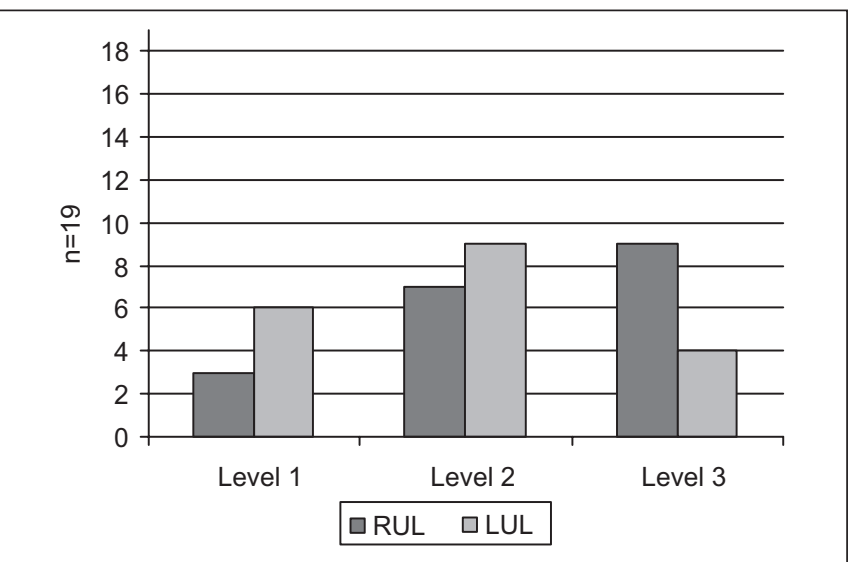

Figure 2 - Upper Limb Motor Block. Grasping Score

Table III - Brachial Motor Block 20 Minutes after Blockade $(n=19)$ *

\begin{tabular}{lccc}
\hline & Level 1 & Level 2 & Level 3 \\
\hline RUL & 3 & 7 & $9^{* *}$ \\
LUL & 6 & 9 & $4^{* *}$ \\
\hline
\end{tabular}

* Measured with mercury sphigmomanometer ERKA $®$, Germany

** Two patients have maintaned digital movement, however weak

As a function of catheter orientation in the epidural space, cranial sensory spread of $\mathrm{T}_{11}-\mathrm{T}_{12}$ has reached or overlapped lower $\mathrm{T}_{2}-\mathrm{T}_{3}$ blockade limits and caudal spread has reached the sacral region. Epidural motor block in $\mathrm{T}_{11}-\mathrm{T}_{12}$ was evaluated by modified Bromage's scale, recording level 1 or partial in 4 patients $(40 \%)$ and level 2 or semi-complete in 6 patients $(60 \%)$ (Figure 3).

Oxymetry and hemodynamic parameters are shown in figures 4,5 and 6 and table IV.

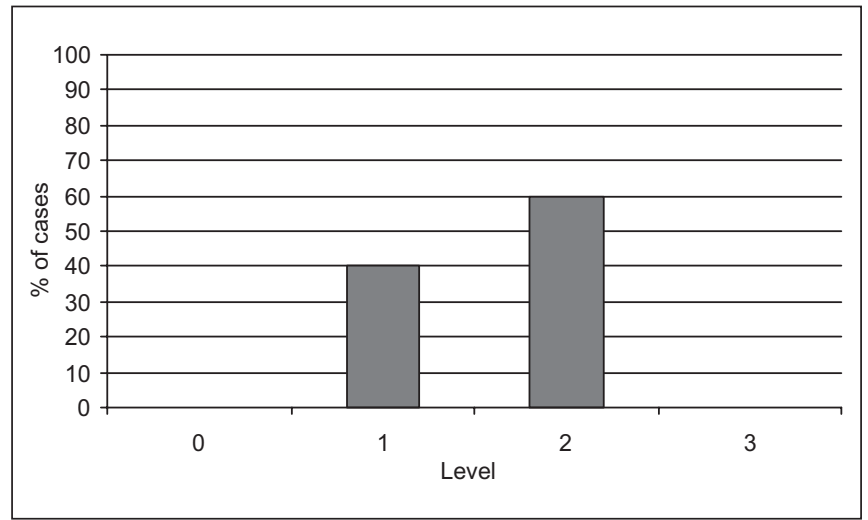

Figure 3 - Lumbosacral Motor Block. Bromage's Scale

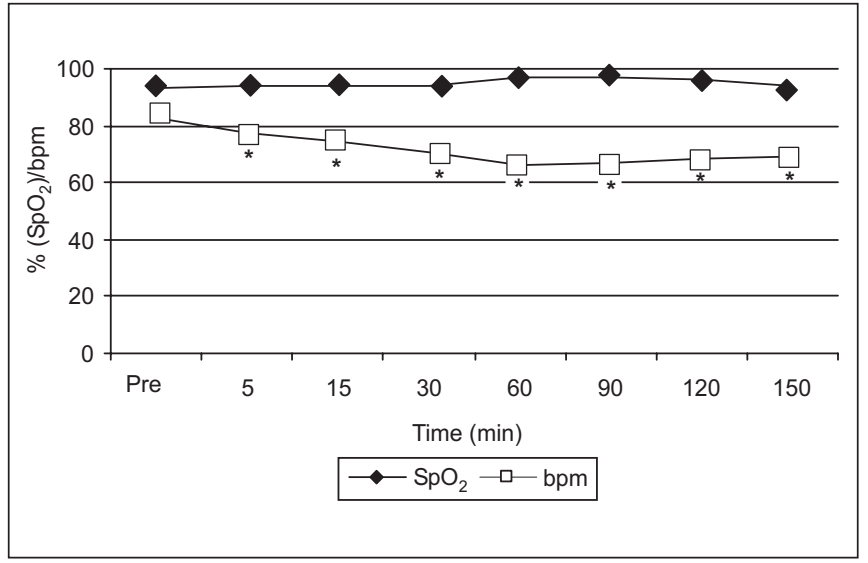

Figure 4 - Mean Perioperative Values of Oxymetry, Heart Rate * Statistically significant as compare to control values

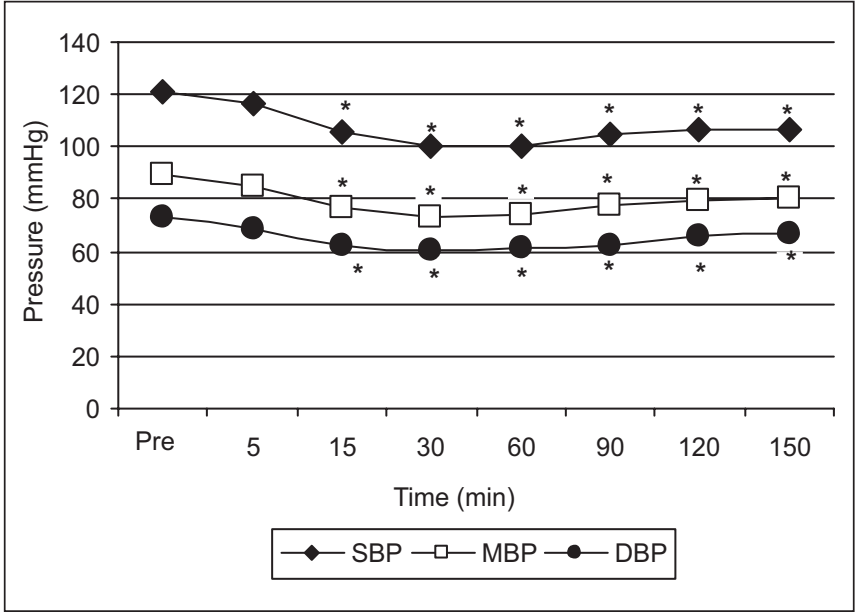

Figure 5 - Mean Perioperative Values, SBP, MBP and DBP * Statistically significant as compare to control values

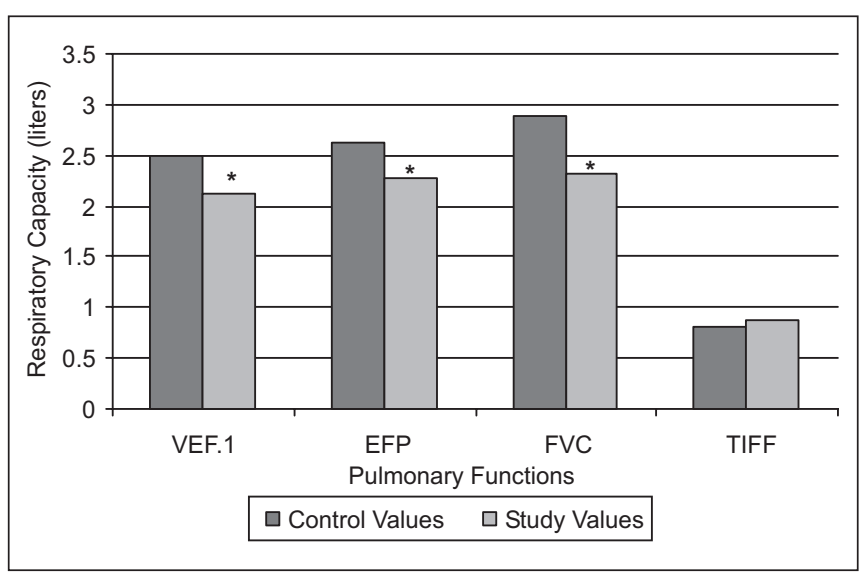

Figure 6 - Spirometric Profile of Respiratory Functions Before and 20 Minutes after Blockade

* Statistically significant as compared to pre-values 
Table IV - Adverse Effects (Patients/\%)

\begin{tabular}{ll}
\hline Claude Bernard Horner's signal & $=4(12.5 \%)$ \\
Bradycardia (rate $\leq 55 \mathrm{bpm})^{*}$ & $=5(15.62 \%)$ \\
Hypotension (SBP $\leq 30 \%$ of control & $=5(15.62 \%)$ \\
or MBP $\leq 50 \mathrm{mmHg} * *$ & \\
Shivering & $=13(40 \%)$ \\
Distress during implants (mammary prosthesis) & $=4(12.5 \%)$ \\
Nausea** / vomiting & $=5 / 0(15.6 \%)$ \\
Pruritus & $=1(3 \%)$ \\
Dyspnea & $=1(3 \%)$ \\
\hline
\end{tabular}

* Treated with $0.5 \mathrm{mg}$ atropine

** Treated with 5 to $15 \mathrm{mg}$ ephedrine

*** Treated with $4 \mathrm{mg}$ ondansetron

\section{Statistical Analysis}

Demographics data are shown in mean, standard deviation and amplitude. Bromage scale data, epidural blocks para-effects and spirometric pulmonary functions test were expressed in relative frequencies using paired Student's $t$ test for variables EFP, VEF1, FVC and VEF1/FVC aiming at evaluating ventilatory parameters changes.

Paired Student's $t$ test was used for Hand Grasping scores before and after $\mathrm{T}_{2}-\mathrm{T}_{3}$ blockade, and ANOVA parametric test (Analysis of Variance) for repeated measures followed by significant minimum differences test were used to compare hemodynamic means (SBP, MBP, DBP and HR). Significance level was $p<0.05$.

\section{DISCUSSION}

In Brazil, epidural block has become popular for outpatient cosmetic mammaplasties with or without sedation. Continuous propofol ${ }^{4}$ or midazolam ${ }^{9}$ with or without fentanyl are titrated to obtain sedation levels in regional anesthesia. Because the level of sleepiness is a marker to determine sedation regardless of its pharmacological etiology, Mackenzie's Sedation Score ${ }^{8}$ was used for midazolam. The level of ideal sedation in regional anesthetic techniques is "conscious sedation" ${ }^{10}$ or level $2^{8}$, especially when patient's cooperation is needed for some research tests.

Notwithstanding chest dermatomes for mammaplasties be located between $T_{1}-T_{5}$, there is the need to include cervical dermatome $\mathrm{C}_{5}$, whose root also participates in the formation of the phrenic nerve. As a function of nervous fibers thickness, regional anesthesia with local anesthetics on the neuraxis is dissociative, being more cranial for $\beta$ fibers (sympathetic), and more caudal for A $\alpha$ fibers (motor) and, in the intermediate position, sensory fibers. Three reasons are considered for such phenomenon: 1) higher motor fibers resistance as compared to sensory fibers; 2) insufficient anesthetic solution spread to reach at least three consecutive Ranvier nodules ${ }^{11}$ of motor roots located within the dural "sleeve" at intervertebral foramens level ${ }^{12}$; and 3) physiological susceptibility of nervous fibers to local anesthetics, as a consequence of sodium and potassium channels density and metabolic activity of ion pumps ${ }^{12}$.

Something similar must have happened with hand motor evaluation involving radial $\left[\mathrm{C}_{5}-\left(\mathrm{T}_{1}\right)\right]$, median $\left[\left(\mathrm{C}_{5}-\mathrm{T}_{1}\right]\right.$ and $\mathrm{ul}-$ nar $\left[C_{7}-\left(T_{1}\right)\right]$ nerves, and with forearm bending through the cutaneous muscle $\left(\mathrm{C}_{5}-\mathrm{C}_{7}\right)$. The act of closing the hand, squeezing or not objects, as well as the ability to bend and extend the elbow, recruit the referred nerves. Grasp exerted on a pressure cuff previously inflated to $40 \mathrm{mmHg}$ of a mercury column, checked before and 20 minutes after blockade installation gives quantitative information about changes in hand motor activity. In general, anesthetized dermatomes do not overlap corresponding miotomes. This is what happens with upper limbs due to peripheral brachial plexus distribution and ropivacaine's pharmacokinetics in the epidural space. Although epidural blocks in $\mathrm{T}_{2}-\mathrm{T}_{3}$ in our study have also recorded loss of sensitivity in $\mathrm{C}_{4}$, cranial anesthetic solution spread in the epidural space has not been uniform as from $\mathrm{C}_{8}$ with different sensory (Figure 1) and motor (Figure 2) block levels. Cranially, as from $\mathrm{C}_{7}$, medullary cervical widening caused by higher neural density aimed at upper limbs, results in epidural space decrease and may influence anesthetic solution spread. In addition to epidural space narrowing, there are other causes ${ }^{14}$ although there are divergences as to the role of "plica mediana dorsalis" ${ }^{15}$, which is the conjunctive band between dura and ligamentum flavum in the epidural space dividing it in one ventral and two postero-lateral compartments ${ }^{16}$. Since ropivacaine has high anesthetic dissociation as compared to bupivacaine, and especially in relation to lidocaine when added to sufentanil of intense segmental lipophylic property, it may asymmetrically impregnate the narrow cervical epidural space. Both were chosen due to these pharmacological properties to obtain good metameric anesthesia with low risk for respiratory depression as compared to hydrophilic morphine.

In addition to diaphragm, sternocleidomastoid $\left(\mathrm{C}_{2}-\mathrm{C}_{3}\right)^{17}$, medium $\left(\mathrm{C}_{3}-\mathrm{C}_{4}\right){ }^{17}$, anterior and posterior $\left(\mathrm{C}_{5}-\mathrm{C}_{8}\right){ }^{18}$ scalene muscles participate in deep breathe. On the other hand, lower intercostal nerves participate on diaphragm innervation ${ }^{17}$. As a function of these uniquenesses, it is supposed that motor medullary integrity of the cervical contingent, including the phrenic nerve, in the presence of motor paralysis of all intercostal muscles, will assure respiratory continuity and vice-versa ${ }^{19,20}$. However, cervical epidural blockade, according to some investigators ${ }^{6,21}$, determines a certain involvement of the phrenic nerve with $25 \%$ decrease in VEF1 and $9 \%$ in $\mathrm{PaO}_{2}$, although without clinical expression. Conversely, Wittich et al. ${ }^{22}$, inducing epidural block in $\mathrm{C}_{7}-\mathrm{T}_{1}$ with $18 \mathrm{~mL}$ of $0.5 \%$ bupivacaine or $1 \%$ mepivacaine, during head and neck surgeries, some of them extending to the chest for oncologic mastectomies, have observed and increase in VEF1 in patients with chronic obstructive pulmonary disease. Similarly, with tracheostomy metamers level $\mathrm{C}_{3}$ and $\mathrm{C}_{4}$ under epidural block in $\mathrm{C}_{7}-\mathrm{T}_{1}$, with loss of sensitivity in $\mathrm{C}_{2}$, with $6 \mathrm{~mL}$ of $1.5 \%$ lidocaine, there has been no spontaneous ventilation decrease ${ }^{22}$. In this patient, "handshaking" test was normal. 
Motor depression of intercostal muscles may be evaluated by coughing ${ }^{24}$, by clinical observation of chest expansion during deep breathe, by magnetometry of forced chest expansion ${ }^{25}$, by electromyography, by arterial blood gas analysis or spirometric test. Total pulmonary capacity is obtained with maximum inspiration and expiration as fast and intense as possible in a portable spirometer to obtain Forced Vital Capacity (FVC). Other pulmonary functions may be evaluated during this expiratory maneuver, such as Forced Expiratory Volume in the first second (FEV1) and Expiratory Flow Peak (EFP). FEV1 is more easily reproduced for being effort-dependent, but EFP is also a good indicator in the initial stage of breathing where internal intercostal muscles $\left(T_{1}-T_{11}\right)$ and abdominal muscles $\left(T_{6}-L_{1}\right)$ have active participation. According to arterial blood gas analysis and spirometric tests, high thoracic epidural block should not be the reason for respiratory distress. Although the sensation of dispnea may ${ }^{5,21}$ or not 26,27 be a consequence of mild hypercapnia, respiratory distress referred by few patients may be a consequence of the excitation of "J" receptors of alveolar walls, as result of blood accumulation or vasodilation by thoracic sympathetic block, especially in dependent regions ${ }^{28}$, or by partial blockade of respiratory muscles motor fibers with decreased respiratory dynamics, phenomenon especially observed in anxious patients.

Most of the times, hemoglobin desaturation depends on respiratory depression promoted by preanesthetic opioids or associated to epidural or spinal anesthetic solutions, changes in chest compliance ${ }^{29}$, tachypnea with low tidal air volume resulting in low effective alveolar ventilation and inhaled $\mathrm{O}_{2}$ concentration. Since patients in our study have received oxygen via nasal catheter during the procedures, values obtained were stable or slightly higher that baseline values (Figure 4). It is worth highlighting that anxiety and sedation may influence oximetry. In fact, this has been observed in some anxious and worried patients who presented tachypnea and initial oximetry values lower than intraoperative values during "conscious" sedation with midazolam.

Under lumbar epidural block, the persistence of abdominal and lower limbs motor activity is relevant during labor and hospital discharge in outpatient procedures, respectively. In our study, we have given priority to motor function in territories reached by epidural block in lower limbs by Bromage score $^{7}$, for being more practical and doable than Van Zundert et al. Score ${ }^{30}$, because according to the latter, as from the supine position, not all patients are able to bend the trunk toward lower limbs.

Muscle shivering during blockade might not have been a sign of bupivacaine toxicity, due to the low dose used, but rather a thermoregulating reflex in an attempt to maintain body temperature as opposed to heat dissipation by peripheral vasodilation of sympathetic block.

Circulatory changes promoted by high thoracic epidural anesthesia are related to sympathetic block which decreases heart rate (negative chronotropism) and contractility (negative inotropism). In relaxed volunteers, plasma catecholamine levels during different epidural blockade levels $\left(T_{8}, T_{4}, C_{8}\right)$ have not recorded significant norepinephrine decrease, suggesting that chemical sympathectomy is only partial ${ }^{31}$ and that compensatory vasoconstriction of other territories not affected by nervous block attenuates blood pressure decrease.

Outpatient setting discharge after epidural block implies motor block recovery of affected limbs without residual instability on upper limbs. Hand motor function evaluation is also a criterium for hospital discharge. However, since there is no quantitative test for upper limbs involvement, subjective "handshaking" test was replaced by an adaptation of Bouaziz et al.'s technique ${ }^{32}$ to determine hand muscle strength. Motor control of lower limbs, responsible for orthostatic posture stability without dizziness, should allow normal ambulation without help. In parallel, the presence of spontaneous micturition, hemodynamic stability, tolerance to food intake, lack of nausea and vomiting, and postoperative analgesia complete discharge criteria ${ }^{33}$.

Two thirds of phrenic roots partially participate on upper limbs plexus formation, especially it if is pre-fixed. Although most authors have not observed possible motor repercussions on upper limbs under high thoracic or cervico-thoracic blockades with decreased anesthetic doses and volumes, these in fact occur, as it has been quantitatively shown in this study by the pressure cuff with mercury column. However, according to preliminary observations and to this study, mild spirometric changes without clinical repercussions are observed under thoracic epidural block, being more a consequence of intercostal nerves paralysis than of phrenic nerve paralysis, resulting in respiratory dynamics preservation.

\section{ACKNOWLEDGEMENTS}

The author acknowledges the epidemiologist Tânia Hirakata and Martin Geier for their help in this study.

\section{REFERÊNCIAS - REFERENCES}

01. Figueiredo RR - Nossa experiência com 1201 casos de anestesia extradural. Rev Bras Cirurgia, 1948;133-152.

02. Gouveia MA, Ribeiro RC - Anestesia peridural cérvico-torácica. Rev Bras Anestesiol, 1974;24:238-248.

03. Leão DG - Peridural torácica: estudo retrospectivo de 1240 casos. Rev Bras Anestesiol, 1997;47:138-147.

04. Nociti JR, Serzedo PSM, Zuccolotto EB et al - Ropivacaína em bloqueio peridural torácico para cirurgia plástica. Rev Bras Anestesiol, 2002;52:156-165.

05. Imbelloni LE - Avaliação da função motora abdominal e parâmetros ventilatórios após peridural torácica. Rev Bras Anestesiol, 1988;38:233-236.

06 . Stevens RA, Frey K, Sheikh T et al - Time course of the effects of cervical epidural anesthesia on pulmonary function. Reg Anesth Pain Med, 1998;23:20-24.

07. Bromage PR - A comparison of the hydrochloride and carbon dioxide salts dioxide salts of lidocaine and prilocaine in epidural analgesia. Acta Anaesthesiol Scand, 1965;16:(Suppl):55-69. 
08. Mackenzie N, Grant IS - Propofol for intravenous sedation. Anaesthesia, 1987;42:3-6.

09. Geier KO, Rocha VHB - Bloqueio contínuo do plexo lombar via compartimento ilíaco, combinado com bloqueio contínuo do nervo femoral em trauma grave de membro inferior. Relato de caso. Rev Bras Anestesiol, 2001;51:53-58.

10. Gwirtz KH - Single-Dose Intrathecal Opioid in the Management of Acute Postoperative Pain, em, Sinatra RS, Hord AH, Ginsgerg B et al - Acute Pain. St Louis. Mosby Year Book, 1992;253-268.

11. Carpenter RL, Mackey DC - Local Anesthetics, em, Barash PG Cullen BF, Stoelting RK - Clinical Anesthesia. Philadelphia. JB Lippincott, 1989;371-403.

12. Fink BR - Mechanism of differential epidural block. Anesth Analg, 1986;65:325-329.

13. Raymond SA, Strichartz GR - The long and short of differential block. Anesthesiology, 1989;70:725-728.

14. Sala-Blanch $X$, Izquierdo E, Fita $G$ et al - Maintained unilateral analgesia. Acta Anaesthesiol Scand, 1995;39:132-135.

15. Asato F, Hirakawa N, Oda M et al - A medium epidural septum is not a common cause of unilateral epidural blockade. Anesth Analg, 1990;71:427-429.

16. Peduto VA, Tani R, Marinelli $L$ et al - Bilateral analgesia and unilateral paresis after lumbar epidural blockade. Anesth Analg, 1992; 74:294-296.

17. Gray H - Anatomia. $29^{a}$ Ed, Rio de Janeiro. Guanabara Koogan, 1977;782-788

18. Netter FH - Atlas of Human Anatomy. Novartis. New Jersey. Ninth Printing, 1997;405

19. Kainuma M, Shimada $Y$, Matsuura M - Cervical epidural anaesthesia in carotid artery surgery. Anaesthesia, 1986;41: 1020-1023.

20. Bonnet F, Derosier JP, Pluskwa F et al - Cervical epidural anaesthesia for carotid artery surgery. Can J Anaesth, 1990;37: 353-358.

21. Takasaki M, Takahashi T - Respiratory function during cervical and thoracic extradural analgesia in patents with normal lungs. Br J Anaesth, 1980;52:1271-1276.

22. Wittich DJ, Berny JJ, Davis RK - Cervical epidural anesthesia for head and neck surgery. Laryngoscope, 1984;94:615-619.

23. Ullman DA, Schmitt L - Tracheostomy performed under cervical epidural blockade. Anesthesiology, 1989;71:161-162.

24. Mineo R, Sharrock NE, Castellano P et al - Effects of adding epinephrine to epidural bupivacaine assessed by thoraco-abdominal muscle strength. Reg Anesth, 1990;15:A1.

25. Urmey WF - Marked distortion of rib cage expansion during epidural anesthesia as measured by magnetometry. Reg Anesth, 1990;15:(Suppl):70.

26. Egbert LD, Tamersoy K, Deas TC - Pulmonary function during spinal anesthesia: the mechanism of cough depression. Anesthesiology, 1961;22:882-885.

27. Askrog VF, Smith TC, Eckenhoff JE - Changes in pulmonary ventilation during spinal anesthesia. Surg Gynecol Obstet, 1964;119:563-567

28. Guyton AC - Tratado de Fisiologia Médica. Rio de Janeiro. Guanabara Koogan, 1988;446-456

29. Novaes MVM, Francisco CRL, Pimenta KB - Estudo comparativo entre bupivacaína a $0,25 \%$ e ropivacaína a $0,2 \%$ em anestesia peridural para cirurgia torácica. Rev Bras Anestesiol, 2001:51:493-502.

30. Van Zundert A, Vaes L, Van der AaP et al - Motor block during epidural anesthesia. Anesth Analg, 1986;65:333-336.
31. Stevens RA, Artuso JD, Kao TC et al - Changes in human plasma catecholamine concentrations during epidural anesthesia depend on the level of block. Anesthesiology, 1991;74: 1029-1034.

32. Bouaziz H, Vial F, Jochum D et al - An evaluation of the cutaneous distribution after obturator nerve block. Anesth Analg, 2002; $94: 445-449$

33. Bello CN - Recuperação pós-anestésica - escalas de avaliação, princípios gerais. CEDAR - Centro de Estudos de Anestesiologia e Reanimação da Disciplina de Anestesiologia da FMUSP, 2000;Ano IV:4-9.

\section{RESUMEN}

Sperhacke D, Geier KO, Eschiletti JCC - Peridural Torácica Alta Asociada o no a la Peridural Torácica Baja en Pacientes Ambulatoriales: Implicaciones Clínicas

JUSTIFICATIVA Y OBJETIVOS: Sobre bloqueo peridural torácico bajo o medio, las alteraciones hemodinâmicas son fácilmente controladas. Como el bloqueo peridural torácico alto $\left(T_{2}-T_{3}\right)$ acomete, frecuentemente, las raíces del plexo braquial $\left(C_{4}\right) C_{5}-T_{1}\left(T_{2}\right)$, algunas de estas responsables por la formación del nervio frénico $\left(\mathrm{C}_{3}-\mathrm{C}_{4}-\mathrm{C}_{5}\right)$ es de suponer, posibles repercusiones motoras de este último. El presente estudio realizado en cirugías estéticas, sobre bloqueo peridural segmentar aislado en $T_{2}-T_{3} O$ asociado al bloqueo peridural segmentar en $T_{11}-T_{12}$, evaluó las repercusiones motoras en la dinámica respiratoria así como en los miembros superiores e inferiores.

MÉTODO: Treinta y dos pacientes, estado físico ASA I y II, sin molestia pulmonar broncoespástica, en actividad y peso corporal igual o superior a $50 \mathrm{~kg}$, fueron sometidos a 21 bloqueos peridurales torácicos aislados en $T_{2}-T_{3}$ y las 11 restantes, a bloqueos peridurales torácicos en $T_{11}-T_{12}$, con ropivacaína a $7,5 \%$ (45 a $90 \mathrm{mg}$ ) asociada al sufentanil (10 a $20 \mu \mathrm{g})$. Repercusiones hemodinámicas, respiratorias y motoras en los miembros superiores e inferiores fueron evaluadas respectivamente, sobre monitorización no invasiva, espirometria, fuerza de preensión de la mano y escala de Bromage.

RESULTADOS: La media de duración de las cirugías mamarias fue de $105 \mathrm{~min}$ con depresión motora de los miembros superiores $(p<0,001)$, con recuperación motora aconteciendo a los 117,2 $\pm 51,3$ min y la primera manifestación de dolor a los $485 \pm 221,2 \mathrm{~min}$. Las cirugías combinadas, que tuvieron una duración media de $165 \mathrm{~min}$, con depresión motora de los miembros inferiores grado 1 , en $40 \%$ y grado 2, en $60 \%$ de los pacientes por la escala de Bromage, cuya recuperación ocurrió $223,9 \pm 57,1$ min y la primera manifestación de dolor en reposo, a los $555 \pm 197,9 \mathrm{~min}$. Las funciones pulmonares, VEF1 (I/seg); PFE (I. $\left.\mathrm{min}^{-1}\right)$; CVF (litros) se presentaron alteradas respectivamente, en 15,20\% ( $p<0,009) ; 13,36 \%$ ( $p<$ $0,029)$ y 18,09\% $(p<0,007)$, con elevación de $8,75 \%$ del VEF1/CVF $(p<0,162)$. Hipotensión arterial $(\leq 30 \%$ de los valores iniciales) y bradicardia $(\leq 55 \mathrm{bpm})$ ocurrieron en cinco pacientes y tremores durante los bloqueos, en trece pacientes.

CONCLUSIONES: Sobre bloqueo torácico alto o cérvicotorácico con dosis y volúmenes reducidos de soluciones anestésicas, ocurren repercusiones motoras en los miembros superiores y en las funciones pulmonares. Entre tanto, a juzgar por observaciones preliminares y del presente estudio, las alteraciones espirométricas fueron estadísticamente significativas, pero, sin expresión clínica en la dinámica respiratoria, siendo esencialmente decurrentes de la parálisis de los nervios intercostales, más que del nervio frénico. 